\title{
Nonlinear Young Integrals via Fractional Calculus
}

\author{
Yaozhong Hu and Khoa N. Lê
}

\begin{abstract}
For Hölder continuous functions $W(t, x)$ and $\varphi_{t}$, we define nonlinear integral $\int_{a}^{b} W\left(d t, \varphi_{t}\right)$ via fractional calculus. This nonlinear integral arises naturally in the Feynman-Kac formula for stochastic heat equations with random coefficients (Hu and Lê, Nonlinear Young integrals and differential systems in Hölder media. Trans. Am. Math. Soc. (in press)). We also define iterated nonlinear integrals.
\end{abstract}

Keywords Nonlinear integration - Young integral • Iterated nonlinear Young integrals

\section{Introduction}

Let $\left\{\varphi_{t}, t \geq 0\right\}$ be a Hölder continuous function and let $\left\{W(t, x), t \geq 0, x \in \mathbb{R}^{d}\right\}$ be another jointly Hölder continuous function of several variables (see (10) for the precise statement about the assumption on $W$ ). The aim of this paper is to define the nonlinear Young integral $\int_{a}^{b} W\left(d t, \varphi_{t}\right)$ by using fractional calculus.

This paper can be considered as supplementary to authors' recent paper [5], where the nonlinear Young integral is introduced to establish the Feynman-Kac formula for general stochastic partial differential equations with random coefficients, namely,

$$
\partial_{t} u(t, x)+L u(t, x)+u(t, x) \partial_{t} W(t, x)=0,
$$

\footnotetext{
Y. Hu $(\varangle) \cdot$ K.N. Lê

Department of Mathematics, University of Kansas, Lawrence, KS 66045, USA e-mail: yhu@ku.edu

K.N. Lê

e-mail:khoale@ku.edu 
where $W$ is a Hölder continuous function of several variables (which can be a sample path of a Gaussian random field) and

$$
L u(t, x)=\frac{1}{2} \sum_{i, j=1}^{d} a_{i j}(t, x, W) \partial_{x_{i} x_{j}}^{2} u(t, x)+\sum_{i=1}^{d} b_{i}(t, x, W) \partial_{x_{i}} u(t, x)
$$

with the coefficients $a_{i j}$ and $b_{i}$ depending on $W$. The terminal condition for the Eq. (1) is $u(T, x)=u_{T}(x)$ for some given function $u_{T}(x)$.

To motivate our study of the nonlinear Young integral, let us recall a basic result in [5] on the Feynman-Kac formula: Let $\sigma(t, x)=\left(\sigma_{i j}(t, x, W)\right)_{1 \leq i, j \leq d}$ satisfy $a(t, x, W)=\sigma(t, x, W) \sigma(t, x, W)^{T}$ (we omit the explicit dependence of $\sigma$ on $W$ ). Consider the following stochastic differential equation

$$
d X_{t}^{r, x}=\sigma\left(t, X_{t}^{r, x}\right) \delta B_{t}+b\left(t, X_{t}^{r, x}\right) d t, \quad 0 \leq r \leq t \leq T, \quad X_{r}^{r, x}=x,
$$

where $\left(B_{t}, 0 \leq t \leq T\right)$ is a standard Brownian motion and $\delta B_{t}$ denotes the Itô differential. Then it is proved in [5] that under some conditions $b$ and $\sigma$ and $W$ (which are verified for certain Gaussian random field $W$ ), the nonlinear integral $\int_{r}^{T} W\left(d s, X_{s}^{r, x}\right)$ is well-defined and exponentially integrable and $u(r, x)=$ $\mathbb{E}^{B}\left\{u_{T}\left(X_{T}^{r, x}\right) \exp \left[\int_{r}^{T} W\left(d s, X_{s}^{r, x}\right)\right]\right\}$ is a Feynman-Kac solution to (1) with $u(T, x)$ $=u_{T}(x)$. One of the main tasks in that paper is the study of the nonlinear Young integral $\int_{r}^{T} W\left(d s, X_{s}^{r, x}\right)$. To this end we used the Riemann sum approximation and the sewing lemma of [2]. In this paper, we shall study the nonlinear Young integral $\int_{a}^{b} W\left(d t, \varphi_{t}\right)$ by means of fractional calculus. This approach may provide more detailed properties of the solutions to the equations (see $[6,7]$ ).

Under certain conditions, we shall prove that the two nonlinear Young integrals, defined by Riemann sums (through sewing lemma) or by fractional calculus, are the same (see Proposition 2).

To expand the solution of a (nonlinear) differential equation with explicit remainder term we need to define (iterated) multiple integrals (see [3]). We shall also give a definition of the iterated nonlinear Young integrals. Some elementary estimates are also obtained.

The paper is organized as follows. Section 2 briefly recalls some preliminary material on fractional calculus that are needed later. Section 3 deals with the nonlinear Young integrals and Sect. 4 is concerned with iterated nonlinear Young integrals.

\section{Fractional Integrals and Derivatives}

In this section we recall some results from fractional calculus.

Let $-\infty<a<b<\infty, \alpha>0$ and $p \geq 1$ be real numbers. Denote by $L^{p}(a, b)$ the space of all measurable functions on $(a, b)$ such that 


$$
\|f\|_{p}:=\left(\int_{a}^{b}|f(t)|^{p} d t\right)^{1 / p}<\infty .
$$

Denote by $C([a, b])$ the space of continuous functions on $[a, b]$. Let $f \in L^{1}([a, b])$. The left-sided fractional Riemann-Liouville integral $I_{a+}^{\alpha} f$ is defined as

$$
I_{a+}^{\alpha} f(t)=\frac{1}{\Gamma(\alpha)} \int_{a}^{t}(t-s)^{\alpha-1} f(s) d s, \quad t \in(a, b)
$$

and the right-sided fractional Riemann-Liouville integral $I_{b-}^{\alpha} f$ is defined as

$$
I_{b-}^{\alpha} f(t)=\frac{(-1)^{-\alpha}}{\Gamma(\alpha)} \int_{t}^{b}(s-t)^{\alpha-1} f(s) d s, \quad t \in(a, b)
$$

where $(-1)^{-\alpha}=e^{-i \pi \alpha}$ and $\Gamma(\alpha)=\int_{0}^{\infty} r^{\alpha-1} e^{-r} d r$ is the Euler gamma function. Let $I_{a+}^{\alpha}\left(L^{p}\right)$ (resp. $\left.I_{b-}^{\alpha}\left(L^{p}\right)\right)$ be the image of $L^{p}(a, b)$ by the operator $I_{a+}^{\alpha}$ (resp. $I_{b-}^{\alpha}$ ). If $f \in I_{a+}^{\alpha}\left(L^{p}\right)$ (resp. $\left.f \in I_{b-}^{\alpha}\left(L^{p}\right)\right)$ and $0<\alpha<1$, then the (left-sided or right-sided) Weyl derivatives are defined (respectively) as

$$
D_{a+}^{\alpha} f(t)=\frac{1}{\Gamma(1-\alpha)}\left(\frac{f(t)}{(t-a)^{\alpha}}+\alpha \int_{a}^{t} \frac{f(t)-f(s)}{(t-s)^{\alpha+1}} d s\right)
$$

and

$$
D_{b-}^{\alpha} f(t)=\frac{(-1)^{\alpha}}{\Gamma(1-\alpha)}\left(\frac{f(t)}{(b-t)^{\alpha}}+\alpha \int_{t}^{b} \frac{f(t)-f(s)}{(s-t)^{\alpha+1}} d s\right) \text {, }
$$

where $a \leq t \leq b$ (the convergence of the integrals at the singularity $s=t$ holds pointwise for almost all $t \in(a, b)$ if $p=1$ and moreover in $L^{p}$-sense if $\left.1<p<\infty\right)$.

It is clear that if $f$ is Hölder continuous of order $\mu>\alpha$, then the two Weyl derivatives exist.

For any $\beta \in(0,1)$, we denote by $C^{\beta}([a, b])$ the space of $\beta$-Hölder continuous functions on the interval $[a, b]$. We will make use of the notation

$$
\|f\|_{\beta ; a, b}=\sup _{a<\theta<r<b} \frac{|f(r)-f(\theta)|}{|r-\theta|^{\beta}}
$$

(which is a seminorm) and

$$
\|f\|_{\infty ; a, b}=\sup _{a \leq r \leq b}|f(r)|,
$$


where $f: \mathbb{R} \rightarrow \mathbb{R}$ is a given continuous function.

It is well-known that $C^{\beta}([a, b])$ with the Hölder norm $\|f\|_{\beta ; a, b}+\|f\|_{\infty ; a, b}$ is a Banach space. However, it is not separable.

Using the fractional calculus, we have (see [9] and also [3])

Proposition 1 Let $0<\alpha<1$. If $f$ and $g$ are continuously differentiable functions on the interval $[a, b]$, then

$$
\int_{a}^{b} f d g=(-1)^{\alpha} \int_{a}^{b}\left(D_{a+}^{\alpha} f(t)\right)\left(D_{b-}^{1-\alpha} g_{b-}(t)\right) d t
$$

where $g_{b-}(t)=g(t)-g(b)$.

In what follows $\kappa$ denotes a universal generic constant depending only on $\lambda, \tau, \alpha$ and independent of $W, \varphi$ and $a, b$. The value of $\kappa$ may vary from occurrence to occurrence.

For two function $f, g:[a, b] \rightarrow \mathbb{R}$, we can define the Riemann-Stieltjes integral $\int_{a}^{b} f(t) d g(t)$. Here we recall a result which is well-known (see for example [3, 9] or $[6,7])$.

Lemma 1 Let $f$ and $g$ be Hölder continuous functions of orders $\alpha$ and $\beta$ respectively. Suppose that $\alpha+\beta>1$. Then the Riemann-Stieltjes integral $\int_{a}^{b} f(t) d g(t)$ exists and for any $\gamma \in(1-\beta, \alpha)$, we have

$$
\int_{a}^{b} f(t) d g(t)=(-1)^{\gamma} \int_{a}^{b} D_{a}^{\gamma} f(t) D_{b-}^{1-\gamma} g_{b-}(t) d t
$$

Moreover, there is a constant $\kappa$ such that

$$
\left|\int_{a}^{b} f(t) d g(t)\right| \leq \kappa\|g\|_{\beta ; a, b}\left(\|f\|_{\infty ; a, b}|b-a|^{\beta}+\|f\|_{\alpha ; a, b}|b-a|^{\alpha+\beta}\right) .
$$

Proof We refer to [9] or [3] for a proof of (8). We shall outline a proof of (9). Let $\gamma$ be such that $\alpha>\gamma>1-\beta$. Applying fractional integration by parts formula (8), we obtain

$$
\left|\int_{a}^{b} f(t) d g(t)\right| \leq \int_{a}^{b}\left|D_{a+}^{\gamma} f(t) D_{b-}^{1-\gamma} g_{b-}(t)\right| d t
$$

From (5) and (6) it is easy to see that

$$
\left|D_{b-}^{1-\gamma} g_{b-}(t)\right| \leq \kappa\|g\|_{\beta ; a, b}(b-r)^{\beta+\gamma-1}
$$


and

$$
\left|D_{a+}^{\gamma} f(t)\right| \leq \kappa\left[\|f\|_{\infty ; a, b}(t-a)^{-\gamma}+\|f\|_{\alpha ; a, b}(t-a)^{\alpha-\gamma}\right] .
$$

Therefore

$$
\begin{array}{r}
\left|\int_{a}^{b} f(t) d g(t)\right| \leq \kappa\|g\|_{\beta ; a, b}\left(\|f\|_{\infty ; a, b} \int_{a}^{b}(t-a)^{-\gamma}(b-t)^{\beta+\gamma-1} d t\right. \\
\left.+\|f\|_{\alpha ; a, b} \int_{a}^{b}(t-a)^{\alpha-\gamma}(b-t)^{\beta+\gamma-1} d t\right) .
\end{array}
$$

The integrals on the right hand side can be computed by making the substitution $t=b-(b-a) s$. Hence we derive (9).

We also need the following lemma in the proofs of our main results.

Lemma 2 Let $f(s, t), a \leq s<t \leq b$ be a measurable function of $s$ and $t$ such that

$$
\int_{a}^{b} \int_{a}^{t} \frac{|f(s, t)|}{(t-s)^{1-\alpha}} d s d t<\infty .
$$

Then

$$
\left.\int_{a}^{b} I_{a+t}^{\alpha, t} f\left(t, t^{\prime}\right)\right|_{t^{\prime}=t} d t=\left.(-1)^{\alpha} \int_{a}^{b} I_{b-}^{\alpha, t^{\prime}} f\left(t, t^{\prime}\right)\right|_{t^{\prime}=t} d t .
$$

Proof An application of Fubini's theorem yields

$$
\begin{aligned}
\left.\int_{a}^{b} I_{a+}^{\alpha, t} f\left(t, t^{\prime}\right)\right|_{t^{\prime}=t} d t & =\frac{1}{\Gamma(\alpha)} \int_{a}^{b} \int_{a}^{t} \frac{f(s, t)}{(t-s)^{1-\alpha}} d s d t \\
& =\frac{1}{\Gamma(\alpha)} \int_{a}^{b} \int_{s}^{b} \frac{f(s, t)}{(t-s)^{1-\alpha}} d t d s \\
& =\left.(-1)^{\alpha} \int_{a}^{b} I_{b-}^{\alpha, t^{\prime}} f\left(t, t^{\prime}\right)\right|_{t^{\prime}=t} d t
\end{aligned}
$$

which is the lemma. 


\section{Nonlinear Integral}

In this section we shall use fractional calculus to define the (pathwise) nonlinear integral $\int_{a}^{b} W\left(d t, \varphi_{t}\right)$. This method only relies on regularity of the sample paths of $W$ and $\varphi$. More precisely, it is applicable to stochastic processes with Hölder continuous sample paths.

Another advantage of this approach is that in the theory of stochastic processes it is usually difficult to obtain almost sure type of results. If the sample paths of the process is Hölder continuous, then one can apply this approach to each sample path and almost surely results are then automatic.

In what follows, we shall use $W$ to denote a deterministic function $W: \mathbb{R} \times \mathbb{R}^{d} \rightarrow$ $\mathbb{R}^{d}$. We make the following assumption on the regularity of $W$ :

(W) There are constants $\tau, \lambda \in(0,1]$ such that for all finite $a<b$ and for all compact sets $K$ of $\mathbb{R}^{d}$, the seminorm

$$
\begin{aligned}
& \quad\|W\|_{\tau, \lambda ; a, b, K} \\
& \quad=\sup _{\substack{a \leq s<t \leq b \\
x, y \in K ; x \neq y}} \frac{|W(s, x)-W(t, x)-W(s, y)+W(t, y)|}{|t-s|^{\tau}|x-y|^{\lambda}} \\
& \quad+\sup _{\substack{a \leq s<t \leq b \\
x \in K}} \frac{|W(s, x)-W(t, x)|}{|t-s|^{\tau}}+\sup _{\substack{a \leq t \leq b \\
x, y \in K ; x \neq y}} \frac{|W(t, y)-W(t, x)|}{|x-y|^{\lambda}},
\end{aligned}
$$

is finite.

About the function $\varphi$, we assume

( $\phi) \varphi$ is locally Hölder continuous of order $\gamma \in(0,1]$. That is, the seminorm

$$
\|\varphi\|_{\gamma ; a, b}=\sup _{a \leq s<t \leq b} \frac{|\varphi(t)-\varphi(s)|}{|t-s|^{\gamma}}
$$

is finite for every $a<b$.

Among the three terms appearing in $(\boldsymbol{W})$, we will pay special attention to the first term. Thus, we denote

$$
[W]_{\tau, \lambda ; a, b, K}=\sup _{\substack{a \leq s<t \leq b \\ x, y \in K ; x \neq y}} \frac{|W(s, x)-W(t, x)-W(s, y)+W(t, y)|}{|t-s|^{\tau}|x-y|^{\lambda}} .
$$

If $a, b$ is clear from the context, we frequently omit the dependence on $a, b$. In addition, throughout the paper, the compact set $K$ can be chosen to be any compact set containing the image of $\varphi$ on the interval of integration. Thus we omit the dependence on $K$ as well. For instance, $\|W\|_{\tau, \lambda}$ is an abbreviation for $\|W\|_{\tau, \lambda ; a, b, K},\|\varphi\|_{\gamma}$ is an 
abbreviation for $\|\varphi\|_{\gamma ; a, b}$ and so on. We shall assume that $a$ and $b$ are finite. Thus it is easy to see that for any $c \in[a, b]$

$$
\sup _{a \leq t \leq b}|\varphi(t)|=\sup _{a \leq t \leq b}|\varphi(c)+\varphi(t)-\varphi(c)| \leq|\varphi(c)|+\|\varphi\|_{\gamma}|b-a|^{\gamma}<\infty .
$$

Thus assumption $(\phi)$ also implies that

$$
\|\varphi\|_{\infty ; a, b}:=\sup _{a \leq t \leq b}|\varphi(t)|<\infty
$$

Remark 1 Given a stochastic process indexed by $(t, x)$, it is possible to obtain almost sure regularity of the type (10) by a multiparameter Garsia-Rodemich-Rumsey inequality. Indeed, this has been explored in [4], see also the last section of [5].

One of our main results in this section is to define $\int_{a}^{b} W\left(d t, \varphi_{t}\right)$ under the condition $\lambda \gamma+\tau>1$ through a fractional integration by parts technique. The following definition is motivated from Lemma 1.

Definition 1 We define

$$
\int_{a}^{b} W\left(d t, \varphi_{t}\right)=\left.(-1)^{\alpha} \int_{a}^{b} D_{a+}^{\alpha, t^{\prime}} D_{b-}^{1-\alpha, t} W_{b-}\left(t, \varphi_{t^{\prime}}\right)\right|_{t^{\prime}=t} d t
$$

whenever the right hand side makes sense.

Remark 2 Assume $d=1$. Let $W(t, x)=g(t) x$ be of the product form and let $\varphi(t)=f(t)$, where $g$ is a Hölder continuous function of exponent $\tau$ and $f$ is a Hölder continuous function of exponent $\lambda$. If $1-\tau<\alpha<\lambda$, then

$$
\begin{aligned}
\int_{a}^{b} W\left(d t, \varphi_{t}\right) & =\left.(-1)^{\alpha} \int_{a}^{b} D_{a+}^{\alpha, t^{\prime}} D_{b-}^{1-\alpha, t} W_{b-}\left(t, t^{\prime}\right)\right|_{t^{\prime}=t} d t \\
& =(-1)^{\alpha} \int_{a}^{b} D_{b-}^{1-\alpha, t} g_{b-}(t) D_{a+}^{\alpha, t} f(t) d t
\end{aligned}
$$

Thus from (8), $\int_{a}^{b} W\left(d t, \varphi_{t}\right)$ is an extension of the classical Young integral $\int_{a}^{b} f(t) d g(t)$ (see [3, 8, 9]). For general $d$, if $W(t, x)=\sum_{b}^{d} g_{i}(t) x_{i}$ and $\varphi_{i}(t)=$ $f_{i}(t)$, then it is easy to see that $\int_{a}^{b} W\left(d t, \varphi_{t}\right)=\sum_{i=1}^{d} \int_{a}^{b} f_{i}(t) d g_{i}(t)$.

The following result clarifies the context in which Definition 1 is justified. 
Theorem 1 Assume the conditions $(\boldsymbol{W})$ and $(\boldsymbol{\phi})$ are satisfied. In addition, we suppose that $\lambda \gamma+\tau>1$. Let $\alpha \in(1-\tau, \lambda \tau)$. Then the right hand side of (11) is finite and is independent of $\alpha \in(1-\tau, \lambda)$. As a consequence, we have

$$
\begin{aligned}
& \int_{a}^{b} W\left(d t, \varphi_{t}\right) \\
& =\left.(-1)^{\alpha} \int_{a}^{b} D_{a+}^{\alpha, t^{\prime}} D_{b-}^{1-\alpha, t} W_{b-}\left(t, \varphi_{t^{\prime}}\right)\right|_{t^{\prime}=t} d t \\
& =-\frac{1}{\Gamma(\alpha) \Gamma(1-\alpha)}\left\{\int_{a}^{b} \frac{W_{b-}\left(t, \varphi_{t}\right)}{(b-t)^{1-\alpha}(t-a)^{\alpha}} d t\right. \\
& +\alpha \int_{a}^{b} \frac{W_{b-}^{t}\left(t, \varphi_{t}\right)-W_{b-}\left(t, \varphi_{r}\right)}{(b-t)^{1-\alpha}(t-r)^{\alpha+1}} d r d t \\
& +(1-\alpha) \int_{a}^{b} \int_{t}^{b} \frac{W\left(t, \varphi_{t}\right)-W\left(s, \varphi_{t}\right)}{(s-t)^{2-\alpha}(t-a)^{\alpha}} d s d t \\
& \left.+\alpha(1-\alpha) \int_{a}^{b} \int_{a}^{t} \int_{t}^{b} \frac{W\left(t, \varphi_{t}\right)-W\left(s, \varphi_{t}\right)-W\left(t, \varphi_{r}\right)+W\left(s, \varphi_{r}\right)}{(s-t)^{2-\alpha}(t-r)^{\alpha+1}} d s d r d t\right\}
\end{aligned}
$$

where $W_{b-}(t, x)=W(t, x)-W(b, x)$. Moreover, there is a universal constant $\kappa$ depending only on $\tau, \lambda$ and $\alpha$, but independent $W, \varphi$ and $a, b$ such that

$$
\left|\int_{a}^{b} W\left(d t, \varphi_{t}\right)\right| \leq \kappa\|W\|_{\tau, \lambda ; a, b}(b-a)^{\tau}+\kappa\|W\|_{\tau, \lambda ; a, b}\|\varphi\|_{\gamma ; a, b}^{\lambda}(b-a)^{\tau+\lambda \gamma},
$$

where $\|W\|_{\tau, \lambda ; a, b}=\|W\|_{\tau, \lambda ; a, b, K}$ and $K$ is the closure of the image of $\left(\varphi_{t}, a \leq\right.$ $t \leq b)$.

Proof We denote $\|W\|=\|W\|_{\tau, \lambda ; a, b}$. First by the definitions of fractional derivatives (5) and (6), we have

$$
D_{b-}^{1-\alpha, t} W_{b-}\left(t, \varphi_{t^{\prime}}\right)=\frac{(-1)^{1-\alpha}}{\Gamma(\alpha)}\left(\frac{W_{b-}\left(t, \varphi_{t^{\prime}}\right)}{(b-t)^{1-\alpha}}+(1-\alpha) \int_{t}^{b} \frac{W\left(t, \varphi_{t^{\prime}}\right)-W\left(s, \varphi_{t^{\prime}}\right)}{(s-t)^{2-\alpha}} d s\right) .
$$


and

$$
\begin{aligned}
& D_{a+}^{\alpha, t^{\prime}} D_{b-}^{1-\alpha, t} W_{b-}\left(t, \varphi_{t^{\prime}}\right) \\
& =\frac{(-1)^{1-\alpha}}{\Gamma(\alpha) \Gamma(1-\alpha)}\left(\frac{1}{\left(t^{\prime}-a\right)^{\alpha}} \frac{W_{b-}\left(t, \varphi_{t^{\prime}}\right)}{(b-t)^{1-\alpha}}+\alpha \int_{a}^{t^{\prime}} \frac{W_{b-}\left(t, \varphi_{t^{\prime}}\right)-W_{b-}\left(t, \varphi_{r}\right)}{\left(t^{\prime}-r\right)^{\alpha+1}(b-t)^{1-\alpha}} d r\right. \\
& +\frac{1-\alpha}{\left(t^{\prime}-a\right)^{\alpha}} \int_{t}^{b} \frac{W\left(t, \varphi_{t^{\prime}}\right)-W\left(s, \varphi_{t^{\prime}}\right)}{(s-t)^{2-\alpha}} d s \\
& \left.+(1-\alpha) \int_{a}^{t^{\prime}} \frac{\alpha}{\left(t^{\prime}-r\right)^{\alpha+1}} \int_{t}^{b} \frac{W\left(t, \varphi_{t^{\prime}}\right)-W\left(s, \varphi_{t^{\prime}}\right)-W\left(t, \varphi_{r}\right)+W\left(s, \varphi_{r}\right)}{(s-t)^{2-\alpha}} d s d r\right) .
\end{aligned}
$$

Thus the right hand side of (11) is

$$
\begin{aligned}
& -\frac{1}{\Gamma(\alpha) \Gamma(1-\alpha)}\left\{\int_{a}^{b} \frac{W_{b-}\left(t, \varphi_{t}\right)}{(b-t)^{1-\alpha}(t-a)^{\alpha}} d t+\alpha \int_{a}^{b} \int_{a}^{t} \frac{W_{b-}\left(t, \varphi_{t}\right)-W_{b-}\left(t, \varphi_{r}\right)}{(b-t)^{1-\alpha}(t-r)^{\alpha+1}} d r d t\right. \\
& +(1-\alpha) \int_{a}^{b} \int_{t}^{b} \frac{W\left(t, \varphi_{t}\right)-W\left(s, \varphi_{t}\right)}{(s-t)^{2-\alpha}(t-a)^{\alpha}} d s d t \\
& \left.+\alpha(1-\alpha) \int_{a}^{b} \int_{a}^{t} \int_{t}^{b} \frac{W\left(t, \varphi_{t}\right)-W\left(s, \varphi_{t}\right)-W\left(t, \varphi_{r}\right)+W\left(s, \varphi_{r}\right)}{(s-t)^{2-\alpha}(t-r)^{\alpha+1}} d s d r d t\right\} \\
& =: I_{1}+I_{2}+I_{3}+I_{4} .
\end{aligned}
$$

The condition $(\boldsymbol{W})$ implies

$$
\begin{aligned}
I_{1} & \leq \kappa\|W\| \int_{a}^{b}(b-t)^{\tau+\alpha-1}(t-a)^{-\alpha} d t \\
& =\kappa\|W\|(b-a)^{\tau} .
\end{aligned}
$$

Similarly, we also have

$$
\begin{aligned}
I_{3} & \leq \kappa\|W\| \int_{a}^{b} \int_{t}^{b}(s-t)^{\tau+\alpha-2}(t-a)^{-\alpha} d s d t \\
& \leq \kappa\|W\|(b-a)^{\tau} .
\end{aligned}
$$


The assumptions $(\boldsymbol{W})$ and $(\boldsymbol{\phi})$ also imply

$$
\begin{aligned}
\left|W_{b-}\left(t, \varphi_{t}\right)-W_{b-}\left(t, \varphi_{r}\right)\right| & \leq \kappa\|W\||b-t|^{\tau}\left|\varphi_{t}-\varphi_{r}\right|^{\lambda} \\
& \leq \kappa\|W\|\|\varphi\|_{\gamma}^{\lambda}|b-t|^{\tau}|t-r|^{\lambda \gamma} .
\end{aligned}
$$

This implies

$$
\begin{aligned}
I_{2} & \leq \kappa\|W\|\|\varphi\|_{\gamma}^{\lambda} \int_{a}^{b} \int_{a}^{t}(b-t)^{\tau+\alpha-1}(t-r)^{\lambda \gamma-\alpha-1} d r d t \\
& \leq \kappa\|W\|\|\varphi\|_{\gamma}^{\lambda}(b-a)^{\tau+\lambda \gamma} .
\end{aligned}
$$

Using

$$
\left|W\left(t, \varphi_{t}\right)-W\left(s, \varphi_{t}\right)-W\left(t, \varphi_{r}\right)+W\left(s, \varphi_{r}\right)\right| \leq \kappa\|W\|\|\varphi\|_{\gamma}^{\lambda}|t-s|^{\tau}|t-r|^{\lambda \gamma},
$$

we can estimate $I_{4}$ as follows.

$$
\begin{aligned}
I_{4} & \leq \kappa\|W\|\|\varphi\|_{\gamma}^{\lambda} \int_{a}^{b} \int_{a}^{t} \int_{t}^{b} \frac{|t-s|^{\tau}|t-r|^{\lambda \gamma}}{(s-t)^{2-\alpha}(t-r)^{\alpha+1}} d s d r d t \\
& \leq \kappa\|W\|\|\varphi\|_{\gamma}^{\lambda}(b-a)^{\tau+\lambda \gamma} .
\end{aligned}
$$

The inequalities (15)-(18) imply that for any $\alpha \in(1-\tau, \gamma \lambda)$, the right hand side of (11) is well-defined. The inequalities (15)-(18) also yield (13).

To show (12) is independent of $\alpha$ we suppose $\alpha^{\prime}, \alpha \in(1-\tau, \lambda \gamma), \alpha^{\prime}>\alpha$. Denote $\beta=\alpha^{\prime}-\alpha$. Using Lemma 2, it is straightforward to see that

$$
\begin{aligned}
& \left.(-1)^{\alpha} \int_{a}^{b} D_{a+}^{\alpha, t} D_{b-}^{1-\alpha, t^{\prime}} W_{b-}\left(t, \varphi_{t^{\prime}}\right)\right|_{t^{\prime}=t} d t \\
& =\left.(-1)^{\alpha} \int_{a}^{b} I_{a+}^{\beta, t} D_{a+}^{\beta, t} D_{a+}^{\alpha, t} D_{b-}^{1-\alpha, t^{\prime}} W_{b-}\left(t, \varphi_{t^{\prime}}\right)\right|_{t^{\prime}=t} d t \\
& =\left.(-1)^{\alpha+\beta} \int_{a}^{b} I_{b-}^{\beta, t^{\prime}} D_{a+}^{\alpha+\beta, t} D_{b-}^{1-\alpha, t^{\prime}} W_{b-}\left(t, \varphi_{t^{\prime}}\right)\right|_{t^{\prime}=t} d t \\
& =\left.(-1)^{\alpha^{\prime}} \int_{a}^{b} D_{a+}^{\alpha^{\prime}, t} I_{b-}^{\beta, t^{\prime}} D_{b-}^{1-\alpha, t^{\prime}} W_{b-}\left(t, \varphi_{t^{\prime}}\right)\right|_{t^{\prime}=t} d t \\
& =\left.(-1)^{\alpha^{\prime}} \int_{a}^{b} D_{a+}^{\alpha^{\prime}, t} D_{b-}^{1-\alpha^{\prime}, t^{\prime}} W_{b-}\left(t, \varphi_{t^{\prime}}\right)\right|_{t^{\prime}=t} d t .
\end{aligned}
$$


This proves the theorem.

Now we can improve the equality (13) as in the following theorem

Theorem 2 Let the assumptions $(\boldsymbol{W})$ and $(\boldsymbol{\phi})$ be satisfied. Let a, $b$, c be real numbers such that $a \leq c \leq b$. Then there is a constant $\kappa$ depending only on $\tau, \lambda$ and $\alpha$, but independent $W, \varphi$ and $a, b, c$ such that

$$
\left|\int_{a}^{b} W\left(d t, \varphi_{t}\right)-W\left(b, \varphi_{c}\right)+W\left(a, \varphi_{c}\right)\right| \leq \kappa\|W\|_{\tau, \lambda ; a, b}\|\varphi\|_{\gamma ; a, b}^{\lambda}(b-a)^{\tau+\lambda \gamma} \text {. }
$$

Proof Let $a \leq c<d \leq b$ and let $\tilde{\varphi}(t)=\varphi(c) \chi_{[c, d)}(t)$, where $\chi_{[c, d)}$ is the indicator function on $[c, d)$. Then

$$
W\left(t, \tilde{\varphi}\left(t^{\prime}\right)\right)= \begin{cases}W(t, \varphi(c)) & c \leq t^{\prime}<d \\ W(t, 0) & \text { elsewhere }\end{cases}
$$

This means $W\left(t, \tilde{\varphi}\left(t^{\prime}\right)\right)=W(t, \varphi(c)) \chi_{[c, d)}\left(t^{\prime}\right)$. Hence, from (8) we have

$$
\begin{aligned}
\int_{a}^{b} W(d t, \tilde{\varphi}(t)) & =\left.(-1)^{\alpha} \int_{a}^{b} D_{b-}^{1-\alpha, t} W_{b-}(t, \varphi(c)) D_{a+}^{\alpha, t^{\prime}} \chi_{[c, d)}\left(t^{\prime}\right)\right|_{t^{\prime}=t} d t \\
& =(-1)^{\alpha} \int_{a}^{b} D_{b-}^{1-\alpha, t} W_{b-}(t, \varphi(c)) D_{a+}^{\alpha, t} \chi_{[c, d)}(t) d t \\
& =W(d, \varphi(c))-W(c, \varphi(c)) .
\end{aligned}
$$

Let $c$ be any point in $[a, b]$. Denote $\tilde{W}(t, x)=W(t, x)-W\left(t, \varphi_{c}\right)$. Then $\tilde{W}$ satisfies $(\boldsymbol{W})$. As in the Eq. (14), we have

$$
\begin{aligned}
\int_{a}^{b} W\left(d t, \varphi_{t}\right)-W\left(b, \varphi_{c}\right)+W\left(a, \varphi_{c}\right) & =\int_{a}^{b} \tilde{W}\left(d t, \varphi_{t}\right) \\
& =\tilde{I}_{1}+\tilde{I}_{2}+\tilde{I}_{3}+\tilde{I}_{4}
\end{aligned}
$$

where $\tilde{I}_{2}=I_{2}$ and $\tilde{I}_{4}=I_{4}$ are the same as $I_{2}$ and $I_{4}$ in the proof of Theorem 1 . But 


$$
\begin{aligned}
& \tilde{I}_{1}=-\frac{1}{\Gamma(\alpha) \Gamma(1-\alpha)} \int_{a}^{b} \frac{W\left(t, \varphi_{t}\right)-W\left(b, \varphi_{t}\right)-W\left(t, \varphi_{c}\right)+W\left(b, \varphi_{c}\right)}{(b-t)^{1-\alpha}(t-a)^{\alpha}} d t \\
& \tilde{I}_{3}=-\frac{(1-\alpha)}{\Gamma(\alpha) \Gamma(1-\alpha)} \int_{a}^{b} \int_{t}^{b} \frac{W\left(t, \varphi_{t}\right)-W\left(s, \varphi_{t}\right)-W\left(t, \varphi_{c}\right)+W\left(s, \varphi_{c}\right)}{(s-t)^{2-\alpha}(t-a)^{\alpha}} d s d t .
\end{aligned}
$$

From the assumptions $(\boldsymbol{W})$ and $(\boldsymbol{\phi})$ we see that

$$
\begin{gathered}
\left|W\left(t, \varphi_{t}\right)-W\left(b, \varphi_{t}\right)-W\left(t, \varphi_{c}\right)+W\left(b, \varphi_{c}\right)\right| \\
\quad \leq \kappa\|W\|_{\tau, \lambda ; a, b}\|\varphi\|_{\gamma ; a, b}^{\lambda}|b-t|^{\tau}|t-c|^{\lambda \gamma} \\
\quad \leq \kappa\|W\|_{\tau, \lambda ; a, b}\|\varphi\|_{\gamma ; a, b}^{\lambda}|b-t|^{\tau}|t-a|^{\lambda \gamma} .
\end{gathered}
$$

This implies that

$$
\tilde{I}_{1} \leq \kappa\|W\|_{\tau, \lambda ; a, b}\|\varphi\|_{\gamma ; a, b}^{\lambda}(b-a)^{\tau+\lambda \gamma} .
$$

Similarly, we have

$$
\tilde{I}_{3} \leq \kappa\|W\|_{\tau, \lambda ; a, b}\|\varphi\|_{\gamma ; a, b}^{\lambda}(b-a)^{\tau+\lambda \gamma} .
$$

Combining these two inequalities (20) and (21) with the inequalities (17) and (18) we have

$$
\left|\int_{a}^{b} \tilde{W}\left(d t, \varphi_{t}\right)\right| \leq \kappa\|W\|_{\tau, \lambda ; a, b}\|\varphi\|_{\gamma ; a, b}^{\lambda}(b-a)^{\tau+\lambda \gamma}
$$

which yields (19).

Theorem 3 Let the assumption $(\boldsymbol{W})$ be satisfied. Let $\varphi:[a, b] \rightarrow \mathbb{R}^{d}$ satisfy

$$
|\varphi(s)-\varphi(a)| \leq L|s-a|^{\ell} \quad \forall s \in[a, b] \quad \text { and } \quad \sup _{a \leq t<s \leq b} \frac{|\varphi(s)-\varphi(t)|}{(s-t)^{\gamma}} \leq L
$$

for some $\ell \in(\gamma, \infty)$ and for some constant $L \in(0, \infty)$. If $\tau+\lambda \gamma>1$, then for any $\beta<1+\frac{\lambda \gamma+\tau-1}{\gamma} \ell$ we have

$$
\left|\int_{a}^{b} W\left(d t, \varphi_{t}\right)-W\left(b, \varphi_{a}\right)+W\left(a, \varphi_{a}\right)\right| \leq C(b-a)^{\beta},
$$

here the constant $C$ does not depend on $b-a$.

Proof As in the proof of Theorem 2 we express $\int_{a}^{b} W\left(d t, \varphi_{t}\right)-W\left(b, \varphi_{a}\right)+W\left(a, \varphi_{a}\right)$ as the sum of the terms $\tilde{I}_{j}, j=1,2,3,4$ (we follow the notation there). First, 
we explain how to proceed with $\tilde{I}_{4}$. We shall use $C$ to denote a generic constant independent of $b-a$. Denote

$$
J:=\left|W\left(t, \varphi_{t}\right)-W\left(s, \varphi_{t}\right)-W\left(t, \varphi_{r}\right)+W\left(s, \varphi_{r}\right)\right|
$$

First, we know that we have

$$
J \leq C|t-s|^{\tau}|t-r|^{\lambda \gamma}
$$

On the other hand, we also have

$$
\begin{aligned}
J & \leq\left|W\left(t, \varphi_{t}\right)-W\left(s, \varphi_{t}\right)-W\left(t, \varphi_{a}\right)+W\left(s, \varphi_{a}\right)\right| \\
& \quad+\left|W\left(t, \varphi_{r}\right)-W\left(s, \varphi_{r}\right)-W\left(t, \varphi_{a}\right)+W\left(s, \varphi_{a}\right)\right| \\
& \leq C|t-s|^{\tau}\left[|t-a|^{\lambda \ell}+|r-a|^{\lambda \ell}\right] \\
\leq & \leq|t-s|^{\tau}|t-a|^{\lambda \ell}
\end{aligned}
$$

when $a \leq r<t<s \leq b$. Therefore, from (24) and (25) it follows that for any $\beta_{1} \geq 0$ and $\beta_{2} \geq 0$ with $\beta_{1}+\beta_{2}=1$, we have

$$
J \leq C|t-s|^{\tau}|t-r|^{\beta_{1} \lambda \gamma}|t-a|^{\beta_{2} \lambda \ell}
$$

If we choose $\alpha$ and $\beta_{1}$ such that

$$
\tau+\alpha>1, \quad \beta_{1} \lambda \gamma-\alpha>0
$$

then

$$
\tilde{I}_{4} \leq C(b-a)^{\beta_{1} \lambda \gamma+\beta_{2} \lambda \ell+\tau} .
$$

For any $\beta<1+\frac{\lambda \gamma+\tau-1}{\gamma} \ell$ we can choose $\alpha, \beta_{1}$, and $\beta_{2}$ such that (26) is satisfied and

$$
\tilde{I}_{4} \leq C(b-a)^{\beta} .
$$

The term $\tilde{I}_{2}$ can be handled in a similar but easier way and a similar bound can be obtained.

Now, let us consider $\tilde{I}_{3}$. We have

$$
\left|W\left(t, \varphi_{t}\right)-W\left(s, \varphi_{t}\right)-W\left(t, \varphi_{a}\right)+W\left(s, \varphi_{a}\right)\right| \leq C|t-s|^{\tau}|t-a|^{\lambda \ell} .
$$

This easily yields

$$
\tilde{I}_{3} \leq C(b-a)^{\tau+\lambda \ell} .
$$

A similar estimate holds true for $\tilde{I}_{1}$. However, it is easy to verify $\tau+\lambda \ell>1+$ $\frac{\lambda \gamma+\tau-1}{\gamma} \ell$ if $\ell>\gamma$. The theorem is proved. 
Next, we show that the nonlinear integral defined in Definition 1 coincides with the limit of Riemann sums. For this purpose, we need some preliminary set up. For every $s, t$ in $[a, b]$, we put $\mu(s, t)=W\left(t, \varphi_{s}\right)-W\left(s, \varphi_{s}\right)$. Let $\pi=\left\{a=t_{0}<t_{1}<\right.$ $\left.\cdots<t_{n}=b\right\}$ be a partition of $[a, b]$ with mesh size $|\pi|=\max _{1 \leq i \leq n}\left|t_{i}-t_{i-1}\right|$. One can consider the limit of the Riemann sums

$$
\lim _{|\pi| \downarrow 0} \sum_{i=1}^{n} \mu\left(t_{i-1}, t_{i}\right)
$$

whenever it exists. A sufficient condition for convergence of the Riemann sums is provided by following two results of [2], see also [1] for a simple exposition.

Lemma 3 (The sewing map) Let $\mu$ be a continuous function on $[0, T]^{2}$ with values in a Banach space $B$ and $\varepsilon>0$. Suppose that $\mu$ satisfies

$$
|\mu(a, b)-\mu(a, c)-\mu(c, b)| \leq K|b-a|^{1+\varepsilon} \quad \forall 0 \leq a \leq c \leq b \leq T .
$$

Then there exists a function $\mathscr{J} \mu(t)$ unique up to an additive constant such that

$$
|\mathscr{J} \mu(b)-\mathscr{J} \mu(a)-\mu(a, b)| \leq K\left(1-2^{-\varepsilon}\right)^{-1}|b-a|^{1+\varepsilon} \quad \forall 0 \leq a \leq b \leq T \text {. }
$$

We adopt the notation $\mathscr{J}_{a}^{b} \mu=\mathscr{J} \mu(b)-\mathscr{J} \mu(a)$

Lemma 4 (Abstract Riemann sum) Let $\pi=\left\{a=t_{0}<t_{1}<\cdots<t_{m}=b\right\}$ be an arbitrary partition of $[a, b]$ with $|\pi|=\sup _{i=0, \ldots, m-1}\left|t_{i+1}-t_{i}\right|$. Define the Riemann sum

$$
J_{\pi}=\sum_{i=0}^{m-1} \mu\left(t_{i}, t_{i+1}\right)
$$

then $J_{\pi}$ converges to $\mathscr{J}_{a}^{b} \mu$ as $|\pi| \downarrow 0$.

Because $\tau+\lambda \gamma$ is strictly greater than 1 , the estimate (19) together with the previous two Lemmas implies

Proposition 2 Assume that $(\boldsymbol{W})$ and $(\boldsymbol{\phi})$ hold with $\lambda \gamma+\tau>1$. As the mesh size $|\pi|$ shrinks to 0 , the Riemann sums

$$
\sum_{i=1}^{n}\left[W\left(t_{i}, \varphi_{t_{i-1}}\right)-W\left(t_{i-1}, \varphi_{t_{i-1}}\right)\right]
$$

converges to $\int_{a}^{b} W\left(d t, \varphi_{t}\right)$.

Remark 3 In [5], the authors define the nonlinear integral $\int W\left(d t, \varphi_{t}\right)$ via the sewing Lemma 3. The previous proposition shows that the approach using fractional calculus employed here produces an equivalent definition. Let us note that this is possible because of the key estimate (19) and the uniqueness part of the sewing Lemma 3. 
It is easy to see from here that

$$
\int_{a}^{b} W\left(d t, \varphi_{t}\right)=\int_{a}^{c} W\left(d t, \varphi_{t}\right)+\int_{c}^{b} W\left(d t, \varphi_{t}\right) \quad \forall a<c<b .
$$

This together with (13) imply easily the following.

Proposition 3 Assume that $(\boldsymbol{W})$ and $(\boldsymbol{\phi})$ hold with $\lambda \gamma+\tau>1$. As a function of $t$, the indefinite integral $\left\{\int_{a}^{t} W\left(d s, \varphi_{s}\right), t \leq a \leq b\right\}$ is Hölder continuous of exponent $\tau$.

Further properties can be developed. For instance, we study the dependence of the nonlinear Young integration $\int W\left(d s, \varphi_{s}\right)$ with respect to the medium $W$ and the integrand $\varphi$. We state the following two propositions whose proofs are left for readers (see, however, [5] for details).

Proposition 4 Let $W_{1}$ and $W_{2}$ be functions on $\mathbb{R} \times \mathbb{R}^{d}$ satisfying the condition $(\boldsymbol{W})$. Let $\varphi$ be a function in $C^{\gamma}\left(\mathbb{R} ; \mathbb{R}^{d}\right)$ and assume that $\tau+\lambda \gamma>1$. Then

$$
\begin{aligned}
\left|\int_{a}^{b} W_{1}\left(d s, \varphi_{s}\right)-\int_{a}^{b} W_{2}\left(d s, \varphi_{s}\right)\right| \leq & \left|W_{1}\left(b, \varphi_{a}\right)-W_{1}\left(a, \varphi_{a}\right)-W_{2}\left(b, \varphi_{a}\right)+W_{2}\left(a, \varphi_{a}\right)\right| \\
& +c\left(\|\varphi\|_{\infty}\right)\left[W_{1}-W_{2}\right]_{\beta, \tau, \lambda}\|\varphi\|_{\gamma}|b-a|^{\tau+\lambda \gamma} .
\end{aligned}
$$

Proposition 5 Let $W$ be a function on $\mathbb{R} \times \mathbb{R}^{d}$ satisfying the condition $(\boldsymbol{W})$. Let $\varphi^{1}$ and $\varphi^{2}$ be two functions in $C^{\gamma}\left(\mathbb{R} ; \mathbb{R}^{d}\right)$ and assume that $\tau+\lambda \gamma>1$. Let $\theta \in(0,1)$ such that $\tau+\theta \lambda \gamma>1$. Then for any $u<v$

$$
\begin{aligned}
& \left|\int_{u}^{v} W\left(d s, \varphi_{s}^{1}\right)-\int_{u}^{v} W\left(d s, \varphi_{s}^{2}\right)\right| \\
& \leq C_{1}[W]_{\tau, \lambda}\left\|\varphi^{1}-\varphi^{2}\right\|_{\infty}^{\lambda}|v-u|^{\tau} \\
& \quad+C_{2}[W]_{\tau, \lambda}\left\|\varphi^{1}-\varphi^{2}\right\|_{\infty}^{\lambda(1-\theta)}|v-u|^{\tau+\theta \lambda \gamma}
\end{aligned}
$$

where $C_{1}$ is an absolute constant and $C_{2}=2^{1-\theta} C_{1}\left(\left\|\varphi^{1}\right\|_{\gamma}^{\lambda}+\left\|\varphi^{1}\right\|_{\gamma}^{\lambda}\right)^{\theta}$.

\section{Iterated Nonlinear Integral}

From Remark 2 we see that if $W(t, x)=\sum_{i=1}^{d} g_{i}(t) x_{i}$ and $\varphi_{i}(t)=f_{i}(t)$, then $\int_{a}^{b} W\left(d t, \varphi_{t}\right)=\sum_{i=1}^{d} \int_{a}^{b} f_{i}(t) d g_{i}(t)$. We know that the multiple (iterated) integrals of 
the form

$$
\int_{a \leq s_{1} \leq s_{2} \leq \cdots \leq s_{n} \leq b} \varphi\left(s_{1}, s_{2}, \ldots, s_{n}\right) d g\left(s_{1}\right) d g\left(s_{2}\right) \cdots d g\left(s_{n}\right)
$$

are well-defined and have applications in expanding the solutions of differential equations (see [3]). What is the extension of the above iterated integrals to the nonlinear integral? To simplify the presentation, we consider the case $d=1$. General dimensions can be considered in a similar way with more complex notations.

We introduce the following notation. Let

$$
\Delta_{n, a, b}:=\left\{\left(s_{1}, \ldots, s_{n}\right) ; a \leq s_{1} \leq s_{2} \leq \cdots \leq s_{n} \leq b\right\}
$$

be a simplex in $\mathbb{R}^{n}$.

Definition 2 Let $\varphi: \Delta_{n, a, b} \rightarrow \mathbb{R}$ be a continuous function. For a fixed $s_{n} \in$ $[a, b]$, we can consider $\varphi\left(\cdot, s_{n}\right)$ as a function of $n-1$ variables. Assume we can define $\int_{\Delta_{n-1, a, s_{n}}} \varphi\left(s_{1}, \ldots, s_{n-1}, s_{n}\right) W\left(d s_{1}, \cdot\right) \cdots W\left(d s_{n-1}, \cdot\right)$, which is a function of $s_{n}$, denoted by $\phi_{n-1}\left(s_{n}\right)$, then we define

$$
\int_{a \leq s_{1} \leq \cdots \leq s_{n} \leq b} \varphi\left(s_{1}, \ldots, s_{n}\right) W\left(d s_{1}, \cdot\right) \cdots W\left(d s_{n}, \cdot\right)=\int_{a}^{b} W\left(d s_{n}, \varphi_{n-1}\left(s_{n}\right)\right) .
$$

In the case $W(t, x)=f(t) x$, such iterated integrals have been studied in [3], where an important case is when $\varphi\left(s_{1}, \ldots, s_{n}\right)=\rho\left(s_{1}\right)$ for some function $\rho$ of one variable. This means that $\varphi\left(s_{1}, \ldots, s_{n}\right)$ depends only on the first variable. This case appears in the remainder term when one expands the solution of a differential equation and can be dealt with in the following way.

Let $F_{1}, F_{2}, \ldots, F_{n}$ be jointly Hölder continuous functions on $[a, b]^{2}$. More precisely, for each $i=1, \ldots, n, F_{i}$ satisfies

$$
\begin{aligned}
& \left|F_{i}\left(s_{1}, t_{1}\right)-F_{i}\left(s_{2}, t_{1}\right)-F_{i}\left(s_{1}, t_{2}\right)+F_{i}\left(s_{2}, t_{2}\right)\right| \\
& \quad \leq\left\|F_{i}\right\|_{\tau, \lambda ; a, b}\left|s_{1}-s_{2}\right|^{\tau}\left|t_{1}-t_{2}\right|^{\lambda}, \text { for all } s_{1}, s_{2}, t_{1}, t_{2} \text { in }[a, b] .
\end{aligned}
$$

We assume that $\tau+\lambda>1$.

Suppose that $F$ is a function satisfying (29) with $\tau+\lambda>1$. The nonlinear integral $\int_{a}^{b} F(d s, s)$ can be defined analogously to Definition 1 . Moreover, for a Hölder continuous function $\rho$ of order $\lambda$, we set $G(s, t)=\rho(t) F(s, t)$, it is easy to see that 


$$
\begin{aligned}
& \left|G\left(s_{1}, t_{1}\right)-G\left(s_{2}, t_{1}\right)-G\left(s_{1}, t_{2}\right)+G\left(t_{1}, t_{2}\right)\right| \\
\leq & \left|\rho\left(t_{1}\right)-\rho\left(t_{2}\right)\right|\left|F\left(s_{1}, t_{1}\right)-F\left(s_{2}, t_{1}\right)\right| \\
& \quad+\left|\rho\left(t_{2}\right) \| F\left(s_{1}, t_{1}\right)-F\left(s_{2}, t_{1}\right)-F\left(s_{1}, t_{2}\right)+F_{i}\left(t_{1}, t_{2}\right)\right| \\
\leq & \left(\|\rho\|_{\tau}\|+\| \rho \|_{\infty}\right)\|F\|_{\tau, \lambda}\left|s_{1}-s_{2}\right|^{\tau}\left|t_{1}-t_{2}\right|^{\lambda} .
\end{aligned}
$$

Hence, the integration $\int \rho(s) F(d s, s)$ is well defined. In addition, it follows from Theorem 2 that the map $t \mapsto \int_{a}^{t} \rho(s) F(d s, s)$ is Hölder continuous of order $\tau$.

We have then easily

Proposition 6 Let $\rho$ be a Hölder continuous function of order $\lambda$. Under the condition (29) and $\tau>1 / 2$, the iterated integral

$$
I_{a, b}\left(F_{1}, \ldots, F_{n}\right)=\int_{a \leq s_{1} \leq \cdots \leq s_{n} \leq b} \rho\left(s_{1}\right) F_{1}\left(d s_{1}, s_{1}\right) F_{2}\left(d s_{2}, s_{2}\right) \cdots F_{n}\left(d s_{n}, s_{n}\right)
$$

is well defined.

In the simplest case when $\rho(s)=1$ and $F_{i}(s, t)=f(s)$ for all $i=1, \ldots, n$, the above integral becomes

$$
\int_{a \leq s_{1} \leq \cdots \leq s_{n} \leq b} d f\left(s_{1}\right) \cdots d f\left(s_{n}\right)=\frac{(f(b)-f(a))^{n}}{n !} .
$$

Therefore, one would expect that

$$
\left|I_{a, b}\left(F_{1}, \ldots, F_{n}\right)\right| \leq \kappa \frac{|b-a|^{\gamma_{n}}}{n !} .
$$

This estimate turns out to be true for (30).

Theorem 4 Let $F_{1}, \ldots, F_{n}$ satisfy (29) and $\rho$ be Hölder continuous with exponent $\lambda$. We assume that $\rho(a)=0$. Denote $\beta=\frac{\lambda+\tau-1}{\lambda}$ and $\ell_{n}=\frac{\beta^{n-1}-1}{\beta-1}+\beta^{n-1}(\tau+$ $\lambda$ ). Then, for any $\gamma_{n}<\ell_{n}$, there is a constant $C_{n}$, independent of a and $b$ (but may depend on $\gamma_{n}$ ) such that

$$
\left|I_{a, b}\left(F_{1}, \ldots, F_{n}\right)\right| \leq C_{n}|b-a|^{\gamma_{n}} .
$$




\section{Proof Denote}

$$
I_{a, s}^{(k)}\left(F_{1}, \ldots, F_{k}\right)=\int_{a \leq s_{1} \leq \cdots \leq s_{k} \leq s} \rho\left(s_{1}\right) F_{1}\left(d s_{1}, s_{1}\right) F_{2}\left(d s_{2}, s_{2}\right) \cdots F_{k}\left(d s_{k}, s_{k}\right) .
$$

Thus, we see by definition that

$$
I_{a, s}^{(k+1)}\left(F_{1}, \ldots, F_{k+1}\right)=\int_{a}^{s} F_{k+1}\left(d r, I_{a, r}^{(k)}\left(F_{1}, \ldots, F_{k}\right)\right)
$$

We prove this theorem by induction on $n$. When $n=1$, the theorem follows straightforwardly from (19) with the choice $c=a$. Indeed, we have $\left|I_{a, t}^{(1)}\right| \leq C|t-a|^{\lambda+\tau}$ and $\left|I_{a, t}^{(1)}-I_{a, s}^{(1)}\right| \leq C|t-s|^{\tau}$.

The passage from $n$ to $n+1$ follows from the application of (23)-(33) and this concludes the proof of the theorem.

Remark 4 The estimate of Theorem 4 also holds true for the iterated nonlinear Young integral $I_{a, b}^{(n)}\left(F_{1}, \ldots, F_{n}\right)=\int_{a \leq s_{1} \leq \cdots \leq s_{k} \leq s} F_{1}\left(d s_{1}, \rho\left(s_{1}\right)\right) F_{2}\left(d s_{2}, s_{2}\right) \cdots$ $F_{n}\left(d s_{n}, s_{n}\right)$, where $I_{a, b}^{(k)}\left(F_{1}, \ldots, F_{k}\right)=\int_{a}^{b} F_{k}\left(d s, I_{a, s}^{(k-1)}\left(F_{1}, \ldots, F_{k-1}\right)\right)$, and $I_{a, b}^{(1)}\left(F_{1}\right)=\int_{a}^{b} F_{1}(d s, \rho(s))$.

Acknowledgments Y. Hu is partially supported by a grant from the Simons Foundation \#209206 and by a General Research Fund of University of Kansas.

Open Access This chapter is distributed under the terms of the Creative Commons Attribution Noncommercial License, which permits any noncommercial use, distribution, and reproduction in any medium, provided the original author(s) and source are credited.

\section{References}

1. Feyel, D., de La Pradelle, A.: Curvilinear integrals along enriched paths. Electron. J. Probab. 11, 860-892 (2006)

2. Gubinelli, M.: Controlling rough paths. J. Funct. Anal. 216, 86-140 (2004)

3. $\mathrm{Hu}, \mathrm{Y}$.: Multiple integrals and expansion of solutions of differential equations driven by rough paths and by fractional Brownian motions. Stochastics 85, 859-916 (2013)

4. Hu, Y., Le, K.: A multiparameter Garsia-Rodemich-Rumsey inequality and some applications. Stochast. Process. Appl. 123, 3359-3377 (2013)

5. Hu, Y., Lê, N.K.: Nonlinear Young integrals and differential systems in Hölder media. Trans. Am. Math. Soc. (in press)

6. Hu, Y., Nualart, D.: Differential equations driven by Hölder continuous functions of order greater than 1/2. In: Benth, F.E. et al. (ed.) Abel Symposium on Stochastic Analysis and Applications, pp. 399-413. Springer (2007) 
7. Hu, Y., Nualart, D.: Rough path analysis via fractional calculus. Trans. Am. Math. Soc. 361, 2689-2718 (2009)

8. Young, L.C.: An inequality of the Hölder type, connected with Stieltjes integration. Acta Math. 67, 251-282 (1936)

9. Zähle, M.: Integration with respect to fractal functions and stochastic calculus. I. Probab. Theory Relat. Fields 111, 333-374 (1998) 